\title{
CERN Upgrade Plans for the LHC and its Injectors
}

\author{
Frank Zimmermann*i \\ CERN, Geneva, Switzerland \\ E-mail: 'frank. zimmermann@cern. ch'
}

The primary goal of CERN and the Large Hadron Collider (LHC) community is to ensure that LHC is operated efficiently, that it achieves nominal performance in the shortest term, and that its performance steadily improves. Since several years the community has been discussing the directions for maximizing the physics reach of the LHC by upgrading the experiments, in particular ATLAS and CMS, the LHC machine and the CERN proton injectors, in a phased approach. The first phase comprises construction of a new proton linac, LINAC4 and an LHC interaction-region (IR) upgrade, with the goal of increasing the LHC luminosity to $2-3 \times 10^{34} \mathrm{~cm}^{-2} \mathrm{~s}^{-1}$, while maximizing the use of mature magnet technologies and of the existing infrastructure. These two projects were approved by the CERN Council in December 2007 and are scheduled for completion in 2014. The second phase foresees further substantial improvements in the injector chain, with a proposed replacement of the aging PS and its booster, by a superconducting proton linac (SPL) and a new higher-energy storage ring, PS2, complemented by modifications in the existing SPS, together with major upgrades of the ATLAS and CMS detectors, and, possibly, including another upgrade of the interaction regions based on a different magnet technology, as well as complementary measures such as crab cavities, or a new beam structure. Completion of this second phase around 2018-2020 should allow further increasing the luminosity of the LHC towards $10^{35} \mathrm{~cm}^{-2} \mathrm{~s}^{-1}$. On an even longer time scale, the magnet technology developed for the second phase could provide the route towards an ultimate energy upgrade of the LHC.

In this report, I first recall a few key challenges inherent in the LHC baseline design, and then describe phased upgrade scenarios for the LHC and its injectors which overcome the design limitations and may ultimately lead to a 10-fold luminosity increase.

European Physical Society Europhysics Conference on High Energy Physics, EPS-HEP 2009,

July 16 - 222009

Krakow, Poland

\footnotetext{
* Speaker.

${ }^{\dagger}$ Studies partially supported by the European Community-Research Infrastructure Activity under the FP7 "Capacities Specific Programme" (EuCARD, Grant Agreement no. 227579).
} 


\section{LHC}

The Large Hadron Collider (LHC) [i, will be the next high-energy frontier machine, succeeding the Tevatron. The LHC proton (and ion) beams will collide in the two high-luminosity experiments of ATLAS and CMS, as well as in two lower-luminosity detectors, ALICE and LHCb. The LHC design center-of-mass energy of $14 \mathrm{TeV}$ is about 7 times the Tevatron's, and its peak luminosity of $10^{34} \mathrm{~cm}^{-2} \mathrm{~s}^{-1}$ about 30 times higher. Reaching the LHC design parameters [1] $\left.{ }_{1}^{1}\right]$ will be challenging, as the latter were greatly pushed in competition with the Superconducting Super Collider formerly under construction in Texas. One challenge is the extremely high stored beam energy, placing stringent requirements on machine protection, on the multi-stage beam collimation, and on mitigation measures for radiation damage, magnet quenches and single-event upsets of electronic devices. Another challenge will be the operation with a large number bunches, 2808 per beam. The latter will give rise to electron-cloud effects (increased heat load inside the SC magnets, degraded vacuum, and possibly beam instabilities), and "parasitic" beam-beam collisions, about 2 times 15 on each side of each primary interaction point (IP), or 120 in total around the ring. To control the impact of the latter, the LHC beams must be collided with a sufficiently large crossing angle so as to separate the beams at the places of parasitic "long-range" encounters by 9-10 $\sigma$. The LHC crossing angle, $\theta_{c}$, introduces a geometric luminosity reduction factor, due to the increase of the effective transverse beam size in the plane of crossing, which for bunches much

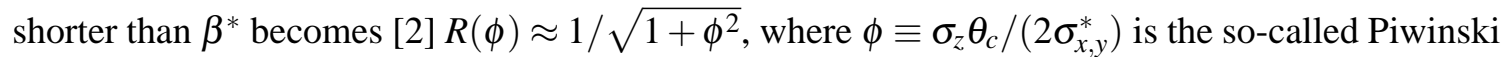
angle, with $\sigma_{z}$ the rms bunch length and $\sigma_{x, y}^{*}$ the transverse rms (round) beam size at the collision point. The nominal LHC operates at $R(\phi) \approx 0.84$; see Fig. $\underline{1}_{1}^{1}$ (left). The reduction factor $R(\phi)$ decreases steeply as $\phi$ is raised beyond nominal, e.g. for smaller $\beta^{*}$ and larger $\theta_{c}$, the Piwinski angle $\phi$ increasing roughly as $1 / \beta^{*}$. Therefore, only reducing $\beta^{*}$ below its nominal value of 55 $\mathrm{cm}$ would bring a rather modest improvement for the nominal separation of $9.5 \sigma$ (Fig. $\underline{1}_{1}^{1}$ (centre)).
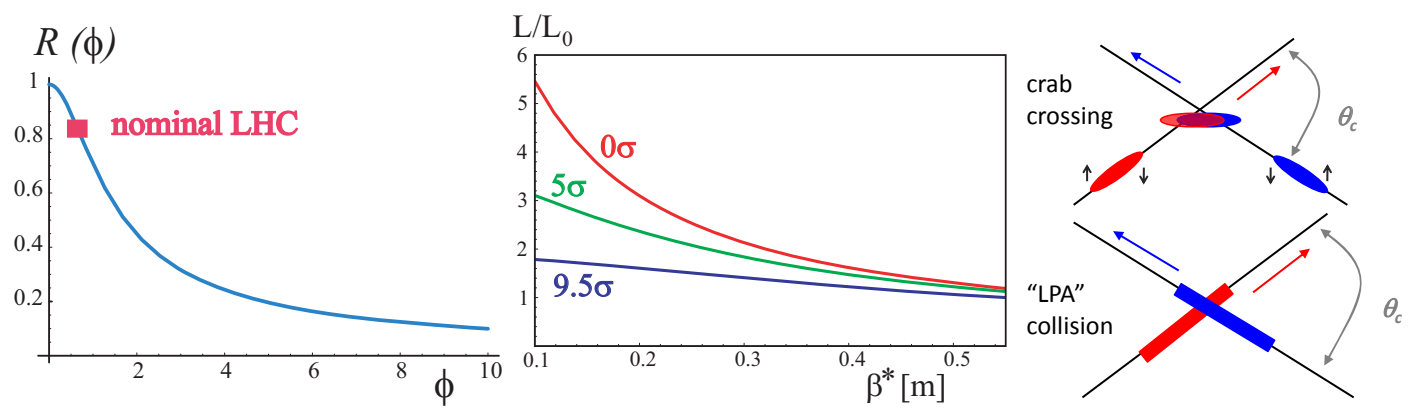

Figure 1: Geometric luminosity reduction factor $R(\phi)$ due to the crossing angle as a function of the Piwinski angle $\phi$; the nominal LHC operating point is also indicated (left). Luminosity $L$ w.r.t. nominal $\left(L_{0}\right)$ versus $\beta^{*}$ for various values of the crossing angle (in units of rms beam divergence) and other parameters equal to their design values (centre). Schematic of crab crossing with $\phi=0$ and "LPA" collision with $\phi \gg 1$ (right).

\section{Super LHC and Beyond}

Since several years, feasibility studies are ongoing for an LHC upgrade with peak luminosity

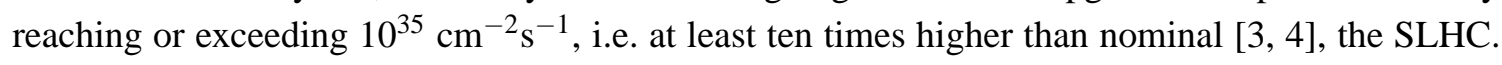


Important issues for the SLHC are the event pile up (number of events per bunch crossing), further decrease of $\beta^{*}$ at the collision points, pertinent new final-focus quadrupoles, higher beam current from new and/or enhanced injectors, an upgrade of the collimation system, magnet lifetimes in a high-radiation environment and associated shielding, and the implied low luminosity lifetime of 2-5 hours. In parallel to the LHC itself, many of the LHC injectors will be replaced by new and more powerful accelerators; see Fig. $\bar{\beta}_{1}$ (left).

Two phases of the SLHC upgrade are foreseen. The LHC Phase-I upgrade consists of new NbTi triplets with larger aperture, new separation dipoles, and a new front quadrupole absorber (TAS), which may allow reaching a $\beta^{*}$ of $0.25-0.30 \mathrm{~m}$ in the IPs 1 and 5 . The beam would be accelerated through the LINAC4, now under construction, providing the "ultimate intensity" of $1.7 \times 10^{11}$ protons per bunch. The SLHC Phase-I upgrade should be completed by 2014, roughly in parallel with the LHC "phase 2" collimation system. SLHC Phase II would be realized around 20182020. It coincides with the commissioning of two new injector-accelerators, the Superconducting Proton Linac (SPL) and the Proton Synchrotron 2 (PS2), which will replace the PS Booster and the aging PS, respectively, and deliver up to twice the ultimate beam brightness with $25 \mathrm{~ns}$ bunch spacing. Rapid cycling synchrotrons or FFAGs have also been proposed as an alternative for (part of) the SPL. In parallel, a coating with low secondary emission yield will be deposited on the vacuum chambers of the existing 6.9-km SPS to suppress the electron cloud. For Phase II the LHC interaction region may need to be rebuilt once again. A promising option is a new triplet made from $\mathrm{Nb}_{3} \mathrm{Sn}$ that might allow squeezing $\beta^{*}$ down to about $15 \mathrm{~cm}$, or below if the final quadrupoles can be shifted closer to the IP, e.g. shifting them from $23 \mathrm{~m}$ to $13 \mathrm{~m}$ might allow for $\beta^{*} \approx 11 \mathrm{~cm}$.

Various upgrade scenarios under consideration are compared with the nominal LHC in Table

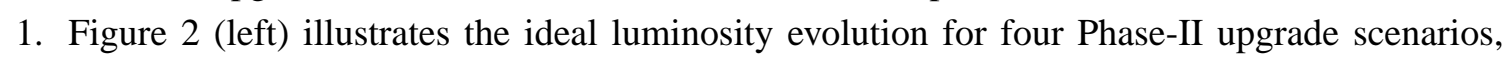
denoted by ES, FCC, LE and LPA. The luminosity for three scenarios (ES, FCC, and LE) starts higher, but decays faster than for the LPA case, leading to shorter runs. The average luminosity values are nearly identical for all four cases. The peak event pile up for the first three options is about $270-310$, or 30-40\% lower than the initially 450 events per crossing for the LPA case.

Luminosity levelling can reduce the maximum event pile up in the detectors (Fig. '2i-i (right)) and the peak power deposited in the IR SC magnets. Three different parameters can be used to level the luminosity: the beam crossing angle, the $\beta^{*}$ and the bunch length. Levelling with the crossing angle has distinct advantages, for example, it reduces the beam-beam tune shift as well as the luminosity, allowing storing more beam current, and rather than decreasing the average luminosity as a levelling through $\beta^{*}$ would do, it increases it if the beam current is not limited by other phenomena, all of which can help to maximize the integrated luminosity.

The Phase-II scenarios are distinguished by their underlying collision schemes. Long-range beam-beam compensation aims at cancelling the de-stabilizing effect of the long-range beam-beam encounters. The principle is to suitably mimic and counteract this interaction by a current carrying wire of opposite effect [i్j], which can be used to reduce the crossing angle. The "early separation scheme" (ES) aims at decoupling the crossing angle at the IP from the beam separation in the common sections by installing dipoles inside the detectors, as close as possible to the IP. The crossing angle can then be further reduced, while still separating the beams as before at most of the parasitic encounters. Full crab crossing (FCC), already used at KEKB, maximizes the overlap of two colliding bunches to be equal to the case of head-on collisions, while the bunch centroids still 

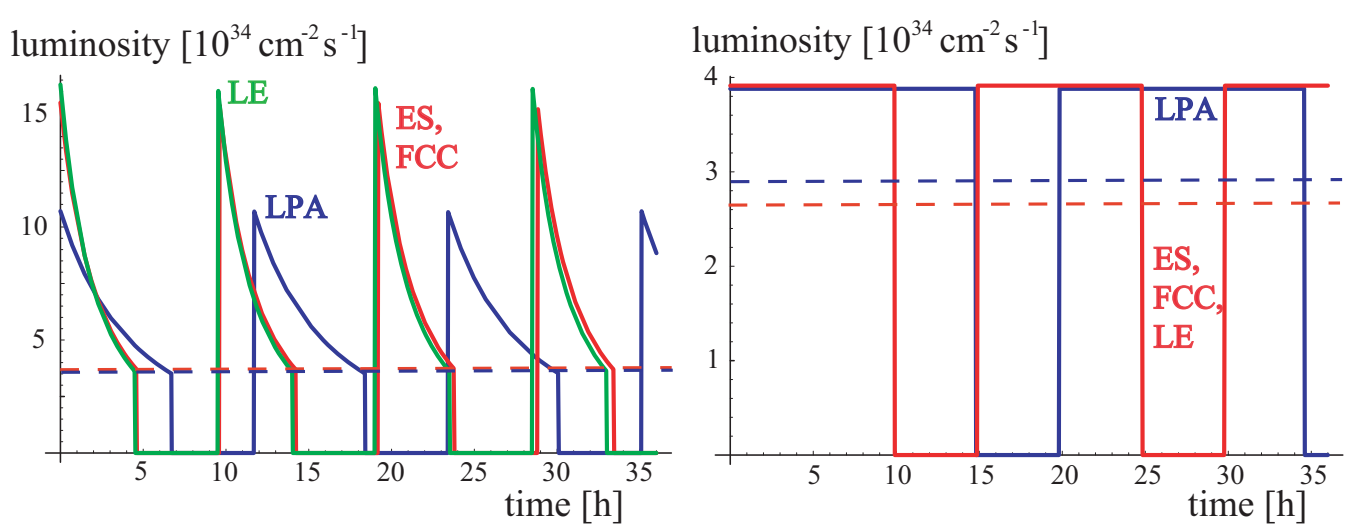

Figure 2: Ideal luminosity evolution for the ES \& FCC (red), LE (green) and LPA scenarios (blue), considering the optimum run duration for a turn-around time of $5 \mathrm{~h}$; the dashed lines indicate the corresponding time-averaged luminosities (left). The same picture with examples of luminosity leveling (right).

cross at an angle. This is achieved via a rotation of the bunches produced by transverse deflecting modes in RF "crab cavities". Alternatively, the luminosity loss from the crossing angle can be recovered by lowering the transverse emittance (LE). In a different spirit, the "Large Piwinski Angle" (LPA) scenario uses a concomitant reduction of the total beam-beam tune shift $\Delta Q_{b b}$, to increase the bunch charge with respect to nominal, as $\Delta Q_{b b}$ cannot much exceed 0.01 according to experience at previous colliders (notably the $\mathrm{S} p \bar{p} \mathrm{~S}$ ). While at low intensity smaller emittance and low Piwinski angle maximize the luminosity, the inverse is true at the beam-beam limit.

Model and prototype SC final-focusing quadrupoles based on $\mathrm{Nb}_{3} \mathrm{Sn}$ are being developed by the US-LARP collaboration, for use in the SLHC Phase II. The SLHC R\&D results also may ultimately enable an increase in the LHC beam energy - the "DLHC" or "TLHC" (LHC with two or three times its present energy). The European Community's EuCARD programme aims for the construction of a first high-field 13- $\mathrm{T} \mathrm{SC} \mathrm{Nb}_{3} \mathrm{Sn}$ dipole, with a 6-T HTS insert, yielding a total field of 19 T, by 2012. Figure '3i' (centre) illustrates past record fields in accelerator-type magnets with an extrapolation to the EuCARD target, as well as the schematic of a TLHC magnet [i, $\bar{i}]$ (right).
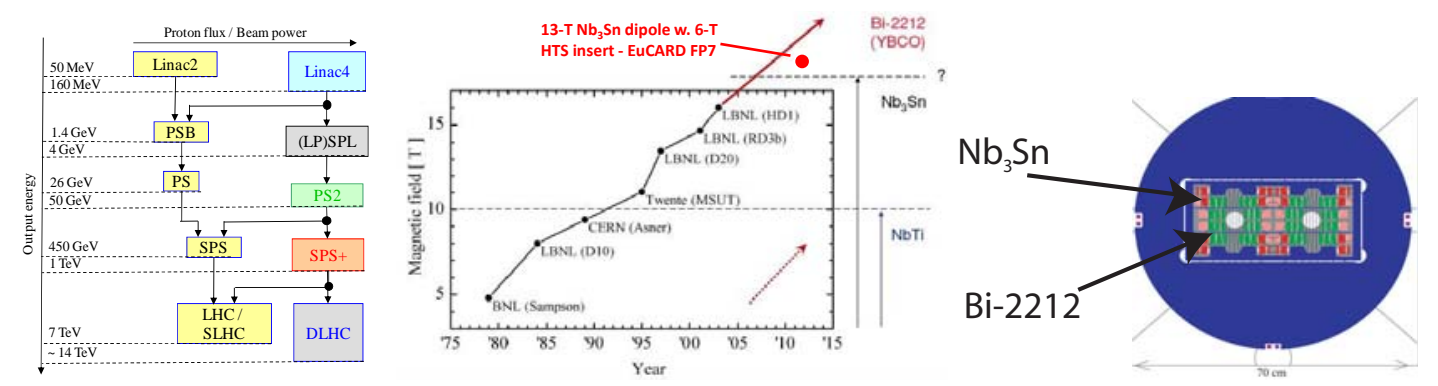

Figure 3: CERN accelerator-complex upgrade plan [י] [left). Field strength increase of SC dipole magnets vs. year with extrapolation [푹] (centre). Schematic of a dual-pipe 24-T block dipole magnet with Bi-2122 in inner high field windings [green] and $\mathrm{Nb}_{3} \mathrm{Sn}$ in outer low field windings [red] [igin] (right).

In summary, several LHC upgrade schemes could raise the peak and average luminosity up to a factor 10 above nominal. Larger-aperture $\mathrm{Nb}_{3} \mathrm{Sn}$ quadrupoles for the LHC interaction regions 
would benefit all options. A parallel rejuvenation of the LHC injector complex will provide beams of higher brightness, minimize the LHC "turnaround time," raise the integrated luminosity, and increase flexibility. A concomitant upgrade of the LHC collimation system appears mandatory. Luminosity leveling becomes a powerful strategy at the highest-luminosity LHC. For the longer term future, stronger dipole magnets and an energy upgrade appear on the horizon.

Table 1: Parameters for the (1) nominal LHC and (2) SLHC Phase-I upgrade, compared with those for four Phase-II upgrade scenarios with (3) more strongly focused ultimate bunches at 25-ns spacing with either early separation and crab cavities [ES] or full crab crossing [FCC], or (4) low emittance [LE], and (5) longer intense flat bunches at 50-ns spacing in a regime of large Piwinski angle [LPA]. The numbers refer to the performance without luminosity leveling. $T_{t a}$ denotes the turnaround time between successive physics runs. The normalized transverse rms emittance is $\gamma \varepsilon=3.75 \mu \mathrm{m}$ in all cases except for LE, with $\gamma \varepsilon=1 \mu \mathrm{m}$.

\begin{tabular}{|lcccccc|}
\hline parameter & symbol & nominal & Phase I & ES or FCC & LE & LPA \\
\hline protons per bunch & $N_{b}\left[10^{11}\right]$ & 1.15 & 1.7 & 1.7 & 1.7 & 4.9 \\
bunch spacing & $\Delta t_{\text {sep }}[\mathrm{ns}]$ & 25 & 25 & 25 & 25 & 50 \\
average current & $I[\mathrm{~A}]$ & 0.58 & 0.86 & 0.86 & 0.86 & 1.22 \\
longitudinal profile & & Gauss. & Gauss. & Gauss. & Gauss. & unif. \\
rms bunch length & $\sigma_{z}[\mathrm{~cm}]$ & 7.55 & 7.55 & 7.55 & 7.55 & 11.8 \\
beta function at IP1\&5 & $\beta^{*}[\mathrm{~m}]$ & 0.55 & 0.30 & 0.08 & 0.10 & 0.25 \\
(effective) crossing angle & $\theta_{c}[\mu \mathrm{rad}]$ & 285 & 410 & 0 & 311 & 381 \\
Piwinski angle & $\phi$ & 0.4 & 1.26 & 0 & 3.2 & 2.01 \\
geometric reduction & & 0.84 & 0.62 & 0.77 & 0.30 & 0.48 \\
peak luminosity & {$\left[10^{34} \mathrm{~cm}^{-2} \mathrm{~s}^{-1}\right]$} & 1.0 & 3.0 & 14.0 & 16.3 & 11.9 \\
events per crossing & & 19 & 57 & 266 & 310 & 452 \\
rms luminous region & {$\left[\mathrm{mm}^{2}\right.$} & 45 & 33 & 53 & 16 & 53 \\
init. luminosity lifetime & $\tau_{\mathrm{L}}[\mathrm{h}]$ & 22 & 11 & 2.2 & 2.0 & 4.0 \\
av. luminosity $\left(T_{\mathrm{ta}}=5 \mathrm{~h}\right)$ & {$\left[10^{34} \mathrm{~cm}^{-2} \mathrm{~s}^{-1}\right]$} & 0.6 & 1.4 & 3.4 & 3.7 & 3.7 \\
\hline
\end{tabular}

\section{References}

[1] O. Brüning et al., LHC Design Report, V.1: the LHC Main Ring, CERN-2004-0030V-1 (2004).

[2] F. Ruggiero, F. Zimmermann, Luminosity Optimization Near the Beam-Beam Limit by Increasing Bunch Length or Crossing Angle, PRST-AB 5, 061001 (2002).

[3] F. Ruggiero (ed.) et al., LHC Luminosity and Energy Upgrade: A Feasibility Study, LHC Project Report 626 (2002).

[4] W. Scandale (ed.), F. Zimmermann (ed.) et al., Proc. Final CARE-HHH Workshop on Scenarios for the LHC Upgrade and FAIR, Chavannes-de-Bogis, CERN-2009-004 (2009).

[5] J.-P. Koutchouk, Principle of a Correction of the Long-Range Beam-Beam Effect in the LHC Using Electromagnetic Lenses, PAC2001, Chicago, 1681 (2001).

[6] R. Garoby, Upgrade Issues for the CERN Accelerator Complex, EPAC'08, Genoa, p. 3734 (2008).

[7] G.L. Sabbi, Wind-and-React Bi-2212 Accelerator Magnet Technology, CARE-HHH WAMSDO workshop, CERN, 19-23 May 2009

[8] P. McIntyre et al., On the Feasibility of a Tripler Upgrade for LHC, PAC'05, Knoxville (2005). 